\title{
Helitrons: Their Impact on Maize Genome Evolution and Diversity
}

\author{
Shailesh K. Lal ${ }^{1}$ and Nikolaos Georgelis ${ }^{2}$ and L. Curtis Hannah ${ }^{2}$ \\ 1 Oakland University, Department of Biological Sciences, lal@oakland.edu \\ 2 University of Florida, Department of Horticultural Sciences, lchannah@ufl.edu
}

\begin{abstract}
Gene movement by the newly-described Helitron family of transposable elements apparently has significantly impacted the evolution of this genome and has contributed to the lack of intra-specific gene collinearity between different maize inbred lines. The abundance of these elements and the extent of diversity among them remain largely undetermined. Several hypotheses have been proposed to explain their transposition and mechanism by which these elements prolifically capture and mobilize gene sequences, but each lacks supporting experimental evidence. A more complete understanding of this process requires molecular and genetic evidence of Helitron activity in modern maize genome. Establishment of in vivo and in vitro systems to assay transposition activity would also aid our understanding of this new class of transposable elements.
\end{abstract}

\section{Introduction and Overview of Helitrons}

Helitron refers to a recently-described class of transposable elements that has a number of important and unique features. Helitrons appear to underlie much of the +/- polymorphisms or "intraspecific violation of genetic colinearity" recently observed in maize genome sequencing projects. This chapter reviews the short documented history of Helitrons and highlights many of the unresolved questions concerning this intriguing class of elements

Helitrons were first hypothesized from computer analysis of the fully sequenced genomes of $C$. elegans, rice and Arabidopsis (Kapitinov and Jurka 2001). These investigators proposed that Helitrons comprise a repetitive class of DNA representing $\sim 2 \%$ of the total genome. An autonomous Helitron was predicted by computer simulation of the consensus sequence of non-autonomous elements. The autonomous element was proposed to contain coding information for a HEL protein composed of a rolling-circle replication initiator and DNA helicase domains needed for transposition. It also contains a replication protein A (RPA)-like protein with single-stranded DNA-binding activity. These proteins are associated with bacterial 
transposons which transpose via rolling circle (RC) replication (Khan 2000; Tavakoli, Comanducci, Dodd, Lett, Albiger, Bennet 2000).

Helitrons are novel because they bear unique characteristics heretofore not shared by known class 1 (transposition through DNA) and class 2 (transposition through RNA) eukaryotic transposable elements. Despite their abundance, Helitrons have remained elusive because of the small size of invariant terminal sequences and the polymorphic nature of internal sequences. The latter characteristic is uniquely attributable to the presence of different gene sequences apparently captured by these elements.

Helitrons lack terminal repeats and do not duplicate host sequence upon insertion. The 5' and 3' terminal ends of Helitrons characterized by Kapitinov and Jurka, (2001) contain sequences TC and CTRR, respectively. They consistently insert between the bases of dinucleotide AT and contain a non-conserved short palindrome sequence located 15-20 nucleotides upstream of their 3' end capable of forming a hairpin structure (Kapitinov and Jurka 2001).

Because an autonomous Helitron was not found from computer searches of the C. elegans, rice and Arabidopsis genomes, there exists the possibility that Helitrons are no longer active in these organisms and the isolated Helitron sequences are simply evolutionary remnants of once-active elements. That Helitrons are active in modern genomes came from three recent reports of mutants caused by Helitron insertions. Two maize mutants, sh2-7527 and bal-ref are caused by insertion of non-autonomous Helitrons (Lal, Giroux, Brendel, Vallejos, Hannah 2003; Gupta, Gallavotti, Stryker, Schmidt, Lal 2005). The mutants likely were caused by relatively recent Helitron insertions since (1) the insertion mutants do not contain any other alteration inactivating the gene (a signature of the evolutionary decay of functional genes) and (2) loss of Sh2 or Bal leads to germination and seed production deficiencies, respectively. Natural selection pressure would significantly limit propagation of these mutants in the absence of human selection. These studies then provided the first evidence that the present-day maize genome harbors an active Helitron. Furthermore, the reports of nearly identical Helitrons inserted in different regions of the maize genome indicate that these elements were active and have moved very recently and may still be active (Lai, Li, Messing, Dooner 2005; Gupta et aA 200105).plant mutant was recently reported in morning glory, Ipomoea tricolor. Isolated in the 1940's, the white flower mutant termed pearly-s-mutant was recently shown to be caused by a Helitron insertion into the dihydroflavonol 4-reductase B gene involved in anthocyanin biosynthesis (Choi, Hoshino, Park, Park, Iada 2007). Intriguingly and unlike maize mutants, this Helitron contains non-functional remnants of sequences encoding helicase and nuclease/ligase.

Helitrons have now been found via sequence analysis in many organisms (reviewed in Kapitonov and Jurka 2007). These include other plants, invertebrates, fungi and fish (Choi et al. 2007; Kapitinov and Jurka 2001; Lal et al. 2003; Poulter, Goodwin, Butler 2003). Massive abundance of Helitrons has been recently reported in a mammalian genome, the brown bat Myotis lucifugus, where they constitute $\sim 3 \%$ of the genome (Pritham and Feschotte 2007). 


\section{Mass Movement of Helitron Mediated Gene Sequences Bestow Intra-specific Violation of Gene Colinearity in Maize.}

Compared to Helitrons in other organisms, maize Helitrons appear unique in their ability to incorporate portions of host gene sequences into the transposable element (reviewed in Kapitinov and Jurka 2007). In doing so Helitrons contribute to the lack of gene colinearity between different maize inbred lines: The maize genome exhibits tremendous diversity which has played a vital role in its domestication. Plant breeders have successfully exploited this diversity for crop improvement. Intriguingly, data emerging from analysis of multiple regions of the genome indicate that significant differences in gene composition and colinearity are widely prevalent among different maize inbred lines. (Fu and Dooner 2002; Song and Messing 2003; Brunner, Pea, Rafalski 2005). This so-called, "intraspecific violation of gene colinearity" or "+/- polymorphism"" has been shown to be primarily due to the presence/absence of gene piece rich fragments between different inbred lines. The lack of gene colinearity between maize lines and the possible contribution of Helitrons to this variation were first reported by Lai et al., 2005 who sequenced the bronze $(b z)$ region of maize inbreds $\mathrm{McC}$ and $\mathrm{B} 73$ and discovered the presence of two gene piece-bearing Helitrons in $\mathrm{McC}$ and their absence in B73. This accounted for the haplotype variability at the $b z$ locus between these two inbred lines. In a parallel study, the extent of lack of gene colinearity between inbred lines B73 and Mo17 was investigated by using oligonucleotide probes (Morgante, Brunner, Pea, Fengler, Zuccolo, Rafalski 2005). Here, $20 \%$ of the detected 20,656 genes were found in only one of the inbred lines. These differences were primarily caused by segments of gene piece-rich insertions rather than deletions among the two lines. Detailed sequence analysis of nine insertions indicated that eight were caused by Helitrons. If this limited data set has any bearing at the genomic level, one would assume that Helitrons may have captured and mobilized thousands of gene pieces across the maize genome. The complete sequencing of maize inbred B73 genome will not provide the complete picture of the extent and diversity of gene piece movement by Helitrons, since Helitron identification and the demarcation of their precise boundaries often entails " $+/$ polymorphism" studies between different inbred lines. Nevertheless, precise identification and annotation of Helitrons is important because the majority of the elements identified thus far represents non-autonomous Helitrons bearing segments of multiple genes. This, no doubt, will complicate the proper annotation of wild type genes in the sequenced maize genome.

\section{Isolated Maize Helitrons Represent a Sub-class of Helitrons.}

Twenty two maize Helitrons displaying +/- polymorphisms have been reported to date (Table 1). Fourteen were found initially through characterization of $+/-$ DNA polymorphisms between maize lines while eight were discovered through sequence similarity to the original 14 elements. Subsequent analysis showed that all eight 


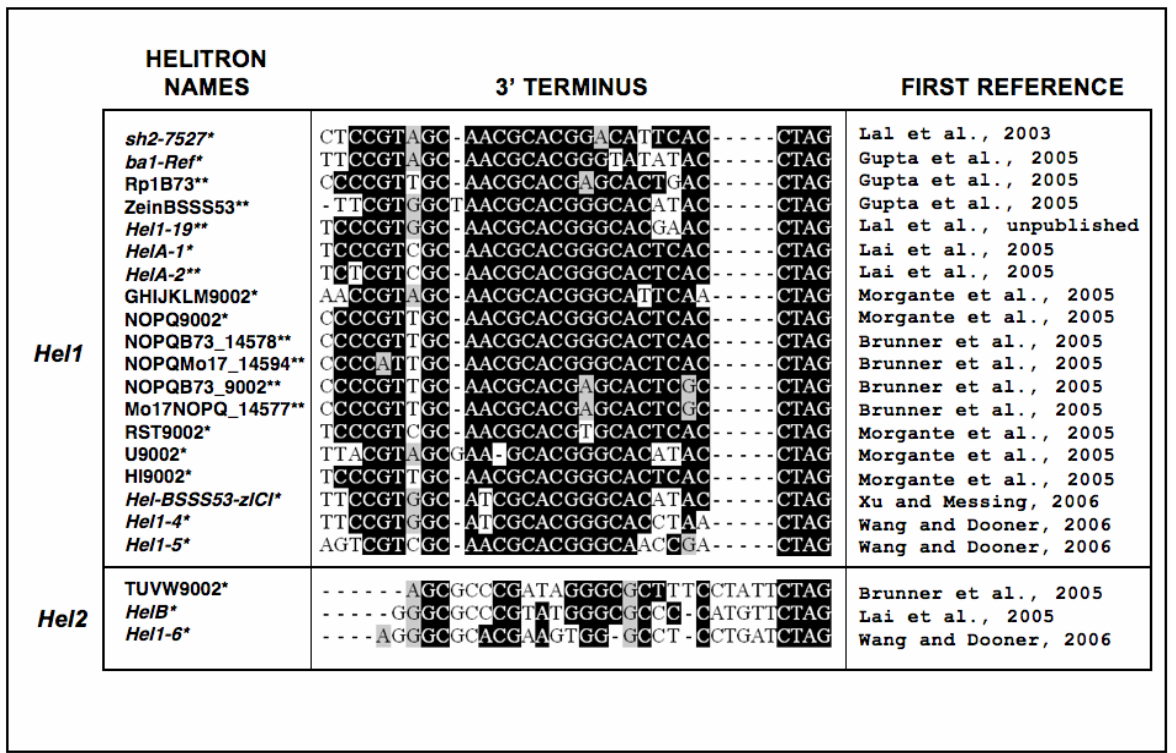

Table. 1. Classification of maize Helitrons based on the conservation of 3' terminal sequence $*$ discovered initially by $+/$ - polymorphism

**discovered by similarity of either terminal or internal sequence to other Helitrons

"derived" elements are associated with $+/$ - polymorphism at their allelic sites or in paralogous genes (references in Table 1). The characterized Helitrons clearly define two closely related families as judged by the similarity of their 3 ' terminal sequence (Table 1). And, placement of gaps in the two families of 3' sequences allows for the detection of relatedness between the two families.

The major sub-family consists of 19 members while the minor group is represented by three Helitrons. As noted above, sequence similarity can be found in the 3' termini and, importantly, relatedness of these maize Helitrons can be seen by the presence of conserved sequences in a 14 bp motif in the 5' termini (data not shown).

While termini of maize Helitrons are strikingly similar, internal sequences exhibit little sequence similarity. Like other transposable elements, the conserved terminal ends of these non-autonomous Helitrons suggest that their movement may be mediated by the transposition protein provided in trans by a master or autonomous member of the family. Searches for candidate autonomous Helitrons have identified several instances in which sequences bearing similarity to DNA helicases are localized in close proximity to Replication protein A, a characteristic of the proposed autonomous Helitron (Gupta et al. 2005; Morgante et al. 2005). However, the presence of premature in-frame stop-codons or large insertions shows that the isolated sequences represent inactive elements. In addition, these putative, mutationallysilenced autonomous elements do not exhibit sequence similarity to the 3 ' conserved terminal ends of isolated maize Helitrons. This suggests that these inactive remnants of proposed autonomous Helitrons may not be closely related to the Helitrons in Table 1. The completion of the B73 genome sequence will determine if this inbred 
harbors a functional autonomous Helitron. However, a functionally autonomous Helitron may not be necessary for Helitron transposition if the helicase and replicase activity are provided by host genes. To date there is no experimental evidence that links the coding sequences of the proposed autonomous Helitrons to transposition activity. In fact there is no direct experimental evidence that helicase and replicase activities are even needed for transposition.

The complete divergence in internal sequence of the characterized maize nonautonomous Helitrons makes it difficult to search for other members in the genome. The highly repetitive nature of genomic DNA hybridizing to Helitron termini (Lal et al. 2003) suggests that there are hundreds to thousands of other family members. Accordingly, the terminal ends of the Helitrons have been used to effectively search for other family members in the maize genome database (Gupta et al. 2005). The scheme used to discover Helitrons is outlined in Fig. 1.

The initial search entails detection in a single clone of sequences highly similar to the Helitron 5' and ' ' termini. The intervening sequence then is viewed as a candidate Helitron. Intervening sequences are then perused for the presence of gene piece-rich sequences since characterized non-autonomous maize Helitrons are rich in gene piece sequences. However, maize Helitrons that do not ferry gene sequences have recently been reported (Wang and Dooner 2006) so the presence of gene sequences within a Helitron is not invariant. Definitive validation that a sequence fulfilling the criteria outlined above is, in fact, a Helitron requires demonstration of +/- polymorphism. One approach, accounting for the isolation of the majority of Helitrons, involves parallel sequencing and comparison of homologous regions in different inbreds. As an alternative, more cost effective strategy, Gupta et al., (2005) searched maize GSS, EST and HTGS clones for sequences aligning with sequences flanking the putative Helitron. Primers were then designed to scan different inbred lines for "empty" sites. This approach has identified 336 putative Helitrons in B73. Of these, 22 exhibit +/- polymorphisms just within the B73 inbred (Lal, unpublished). Since B73 is an inbred, the $+/$ - polymorphism here likely reflects comparison of paralogous loci.

\section{The Mechanism by Which Helitrons Capture and Mobilize Gene Sequences Remains Unknown}

While the occurrence of host gene fragments embedded within transposable elements has been reported in the past it was generally regarded as a rare incidence. However, a recent report of mass movement of gene sequences by a Mutator like family of transposable elements in rice termed MULEs indicates that this process is far more prevalent than initially anticipated (Jiang, Bao, Zhang, Eddy, Wessler 2005). This may play a major role in genome evolution. The MULE family of elements has captured and mobilized more than 1000 gene fragments in the rice genome (Jiang et al., 2005). However, the mechanism by which MULE and Helitron super families of transposable elements acquire gene fragments may differ. For example, $20 \%$ of the gene fragments captured by MULEs bear no apparent wild-type counterpart in the rice genome but display strong similarity to intact genes from other plants. This suggests that the host gene was destroyed during the acquisition process. In contrast, Morgante et al (2005) demonstrate that the wild type progenitor of four different gene fragments captured by a Helitron exist in the maize genome. These investiga- 


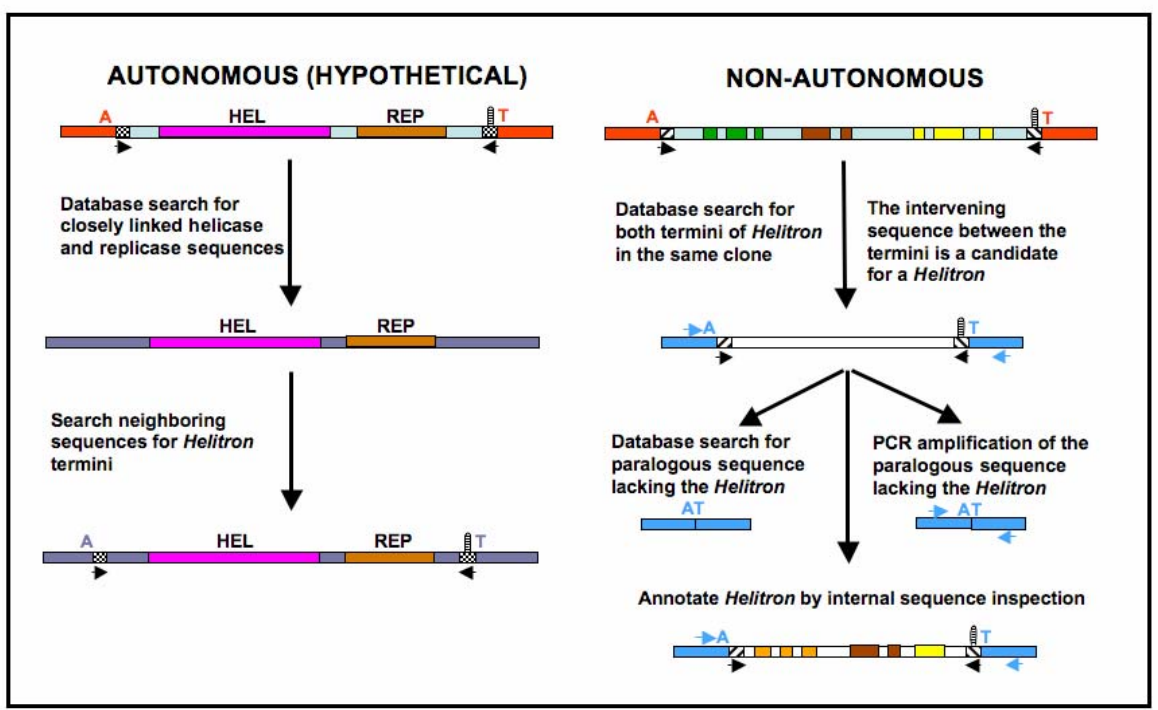

Fig. 1. Strategy for Discovering Maize Helitrons: The upper left and right panels display the structure of autonomous and non-autonomous maize Helitrons. An autonomous Helitron is predicted by computational analysis of related repeat sequences in the genomes of Arabidopsis, rice and Caenorhabditis elegans (Kapitinov and Jurka, 2001). The coding regions of DNA helicase (HEL) and single stranded DNA binding protein (REP) of an autonomous Helitron are marked. The exons captured by the non-autonomous Helitron are displayed by colored blocks. The terminal ends of the Helitron are displayed by pattern filled boxes and the loop near the 3' terminus represents the palindrome sequence. The A and T nucleotide immediately flanking the insertion site of the Helitron are indicated.

tors speculate that Helitron transposition involves replication and strand replacement that preserves the integrity of the donor host sequence. On the other hand, the mechanism of gene capture may not differ for the two types of elements. Given that only one of the two alleles of a gene participates in the events of gene capture, alteration in one allele (and not in the other) creates a heterozygous condition. Given heterozygosity and assuming no selection, it would simply be random chance whether the mutant, non-functional allele or the wildtype allele becomes fixed in the population following gene capture.

The similarity of Helitron transposition proteins, helicase and nuclease/ligase, to host genes led Kaptinov and Jurka (2001) to postulate that these genes also were recruited by the Helitron from the host genome. Further understanding of this process will require understanding of the mechanism by which these elements capture and mobilize host gene sequences.

While Helitron gene piece capture has been reported in several organisms, Helitrons of maize are clearly the most prolific at incorporating gene pieces. Several hypotheses have been forward to explain the mechanism of gene piece capture by 
Helitrons (reviewed in Kapitinov and Jurka 2007). For example, it was proposed that transposition is initiated at the $5^{\prime}$ end of the element and the inefficient recognition of the 3' palindrome sequence as a termination signal by the transposition machinery results in the capture of 3' flanking host sequences (Feschotte and Wessler 2001). The strong conservation of the $3^{\prime}$ end and not the $5^{\prime}$ end of a family of nonautonomous Helitrons led Morgante et al. (2005) to propose that transposition is initiated at the 3' end of the Helitrons and the capture of 5' host flanking sequence occurs during transposition via an unknown mechanism. Kapitinov and Jurka (2007) have proposed that gene piece incorporation occurs through double stranded breaks within the transposing Helitron and the random incorporation of "filler" DNA coming from the host genome.

\subsection{Helitrons and Bacterial Integrons Share a Number of Common Features}

A number of parallels between Helitrons and bacterial integrons suggests that integrons may be the bacterial counterpart of Helitrons (Fig. 2). Like Helitrons, integrons are mobile, lack terminal repeats and do not make host duplications upon insertion. Like Helitrons, integrons capture genes (Hall and Callis 1995). Integrons have the capability to acquire gene sequences from mobile elements called gene cassettes via site specific recombination involving circular intermediates. Analogous to Helitrons, bacterial integrons consist of conserved terminal sequences encompassing a variable region primarily consisting of captured gene cassettes. The captured gene cassettes, like gene pieces in Helitrons are oriented in the same direction. This allows them to be transcribed by the same promoter located in the 5' conserved sequence. Also like Helitrons, different gene cassettes captured by integrons are often co-transcribed as a single unit. In integrons, the site specific recombination during gene capture is mediated by an integrase gene located in the conserved 5' terminus. Whether Helitrons capture gene sequences using mechanisms similar to integrons needs further investigation; however sequences bearing similarity to integrase have not been reported to date in maize Helitrons (Gupta et al. 2005).

\subsection{Potential Role of Helitrons in Maize and Gene Evolution and their Impact on Host Gene Expression}

Integron capture of genes for antibiotic resistance in bacteria has been noted in a number of cases. Expression of the captured gene then provides an obvious advantage to bacteria exposed to the toxin. In contrast, no clear cut example of Helitron gene capture conditioning advantages to the Helitron or to the host organism is available. In fact, all characterized Helitron-captured maize genes are truncated and likely non-functional (Lal et al. 2003; Gupta et al. 2005, Lai et al. 2005; Morgante et al. 2005; Brunner et al. 2005). Helitron capture of an almost intact copy of the maize gene encoding cytidine deaminase was recently reported (Xu and Messing, 2006).

The captured gene lacks its promoter, exon 1 and a major portion of intron 1. While the truncated open reading frame and the introns have been conserved, the lack of detectable transcript of this gene suggests that the gene may not be active. While no a priori reason exists for the absence of intact gene capture in maize, their nonexistence in extant databases is curious. Intact gene capture is possible, at least in 

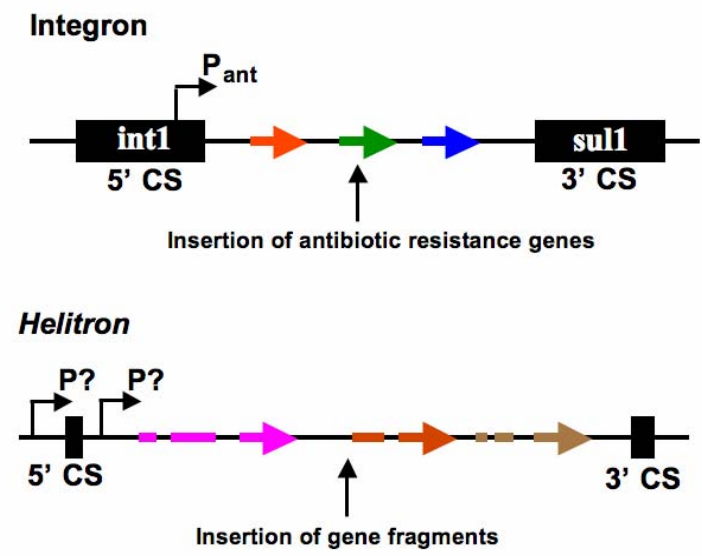

Fig. 2. General structural similarity between Class 1 integrons and gene carrying nonautonomous maize Helitrons: The upper and lower panels display the basic structure of a Class 1 integron and a maize non-autonomous Helitron respectively. The letter P marks the position of the promoter. The 5' and 3' conserved terminal sequences (CS) are indicated. The integrase (int1) and sulfonamide resistance (sul1) are also indicated in the integron structure. The color coded arrows display the coding sequences and direction of the captured genes.

Aspergillus nidulans (Cultrone, Dominguez, Drevet, Scazzocchio, Fernandez-Martin 2007).

The expression of captured genes by Helitrons can potentially have significant impact on host genome expression. For example, the $w p$ mutation in soybean is caused by an insertion of Tgm-Express 1 transposon in the flavnone-3- hydroxylase gene. Similar to Helitrons, Tgm-Express 1 contains fragments of different genes which are transcribed giving rise to multiple, alternatively-spliced, chimeric transcripts. Intriguingly, the $w p$ mutant also displays a significant increase in both seed weight and protein content. Since the genes pieces captured by Tgm-Express 1 represent enzymes involved in diverse metabolic pathways, it was postulated that their transcript may somehow modulate the expression of their wild type genes causing pleiotropic effect of the $w p$ mutant (Zabala and Vodkin, 2005 Zabala and Vodkin 2007).

A potentially significant role for Helitrons is the synthesis of new genes. The gene pieces captured by Helitrons are often transcribed into eclectic transcripts conjoining coding regions of several different genes. These chimeric transcripts are sometimes alternatively spliced, generating several different transcripts via differential utilization of splice sites during pre-mRNA processing (Lal et al. 2003; Morgante et al. 2005; Brunner et al. 2005). For example, the Helitron insertion in maize mutant, sh2-7527 generates several, alternatively-spliced isoforms, at least two of which are caused by alternative retention of a wild type Sh2 intron (Lal et al. 2003). Since 
mutations acting from a distance are known to influence splice site recognition, it is possible that change in the context of captured gene fragments induces alternatively splicing events (Lal et al., 1999).

Promoters located inside or outside Helitrons can initiate transcription. Expression of the sh2-7527 gene uses the Sh2 promoter to produce a chimeric transcript containing sequences of several genes. In contrast, transcription initiation of captured genes within a Helitron has been suggested (Morgante et al., 2005; Brunner et al., 2005). Similarly, transcription of a maize cytochrome P450 monoxygenase captured by a Helitron seems to be initiated inside the element (Lal and Hannah, unpublished results). In this regard, Helitrons parallel pack-MULEs since transcription initiation within many pack-MULEs has been documented (Jiang et al., 2004). The possibility that genes captured by Helitrons may lead to evolution new genes by promoter shuffling was recently reported of xanthine $\alpha$-ketoglutarate-dependent dioxygenase (xanA) gene captured by Helitron-N1_AN in Aspergillus nidulans (Cultrone et al., 2007). Apparently, the 5' region of this gene including its tightly regulated promoter is composed of a Helitron. It is proposed that the 3' portion of a Helitron inserted upstream to the ancestral was deleted and the rolling circle replication initiating from the intact 5' end of the Helitron provided read-through, which terminated in the coding sequence of the downstream xanA gene.

\subsection{Future Prospects and Overview}

The newly discovered Helitrons constitute a vastly unexplored family of novel transposable elements. Observations to date suggest that maize contains a particular subfamily of Helitrons. In contrast to a "generic" Helitron, the maize members bear much sequence similarity in termini. This feature will greatly aid in the quick identification of Helitrons in large-scale sequencing projects. Maize Helitrons also appear to be unique in their ability to prolifically capture gene pieces.

Existing data point to two fundamentally different ways that Helitrons could significantly alter the maize genome and gene expression. First, the ability to capture genes provides a simple mechanism to alter gene dosage. The presence of a gene in one inbred and its absence at the homologous site in another inbred provides an uncomplicated explanation for heterosis. So far however, no case of complete gene capture has been documented in maize so the gene dosage/heterosis model awaits validation. The availability of complete maize genome sequence in the near future will provide a valuable resource to investigate whether Helitrons mediate the mobilization of active genes, and if they do, whether it has occurred to an extent that might impact heterosis.

Second, the ability to place segments of different genes into a common transcribed region provides the opportunity to create new genes and functions. While this aspect of Helitron function awaits validation, it does suggest that "hybrid" transcripts will be found in large EST collections. Studies are in progress.

Despite strong evidence that these elements have moved recently, there is no direct genetic proof of their activity in the present day maize genome. Analysis of Helitron movement in the two maize mutants caused by Helitron insertion may provide clues concerning mechanisms of transposition. Also, a more complete understanding of the process by which two short conserved terminal ends of maize nonautonomous elements mediate movement and integration of large and diverse seg- 
ments of DNA may aid in designing novel strategies for transformation and transgenic expression for crop improvement.

\subsection{Acknowledgments}

The study was supported by NSF grant 0514759 to SL and NSF grants IBN9982626 and 0444031 to LCH and USDA Competitive Grants. 2000-01488, 200603034 and 2007-03575 to LCH. We thank Hugo Dooner for many interesting and fruitful discussions.

\section{References}

Brunner, S., Fengler, K., Morgante, M., Tingey, S. and Rafalski, A. (2005) Evolution of DNA sequence nonhomologies among maize inbreds. Plant. Cell. 17, 343-360.

Brunner, S., Pea. G. and Rafalski, A. (2005) Origins, genetic organization and transcription of a family of non-autonomous Helitron elements in maize. Plant J. 43, 799-810.

Choi, J.D., Hoshino, A., Park, K.I., Park, I.S. and Iida, S. (2007) Spontaneous mutations caused by a Helitron transposon, Hel-It1, in morning glory, Ipomoea tricolor. Plant. J. 49, 924-34.

Cultrone, A., Dominguez, Y.R., Drevet, C., Scazzocchio, C. and Fernandez-Martin, R. (2007) The tightly regulated promoter of the xanA gene of Aspergillus nidulans is included in a Helitron. Mol. Microbiol.63, 1577-87.

Feschotte, C. and Wessler, S.R.(2001) Treasures in the attic: Rolling circle transposons discovered in eukaryotic genomes. Proc. Natl. Acad. Sci. USA. 98, 8923-8924.

$\mathrm{Fu}, \mathrm{H}$. and Dooner, H.K. (2002) Intraspecific violation of genetic colinearity and its implication in maize. Proc. Natl. Acad. Sci. USA. 99, 9573-9578.

Gupta, S., Gallavotti, A., Stryker, GA., Schmidt, R.J. and Lal, S.K. (2005) A novel class of Helitron-related transposable elements in maize contain portions of multiple pseudogenes. Plant. Mol. Biol. 57, 115-27.

Hall, R.and Callis, C. (1995) Mobile gene cassettes and integrons: capture and spread of genes by site specific recombination. Mol. Micro. 15, 593-600.

Jiang, N., Bao, Z., Zhang, X., Eddy, S.R. and Wessler S.R. (2004) Pack-MULE transposable elements mediate gene evolution in plants. Nature. 431:569-73.

Kapitonov, V.V. and Jurka, J. (2001) Rolling-circle transposons in eukaryotes. Proc. Natl. Acad. Sci. USA. 98, 8714-9.

Kapitonov, V.V. and Jurka, J. (2007) Helitrons on a roll: eukaryotic rolling-circle transposons. Trends. Genet. (In press)

Khan, S.A. (2000) Plasmid rolling circle replication: recent development. Mol Microl. 37, 477-484.

Lai, J., Li, Y., Messing, J. and Dooner, H.K. (2005) Gene movement by Helitron transposons contributes to the haplotype variability of maize. Proc. Natl. Acad. Sci. U S A. 102, 906873.

Lal, S.K., Giroux, M.J., Brendel, V., Vallejos, C.E. and Hannah, L.C. (2003) The maize genome contains a Helitron insertion. Plant. Cell. 15, 381-91.

Lal, S.K., Choi, J.H., Shaw, J. and Hannah, L.C. (1999) A splice site mutant of maize activates cryptic splice sites, elicits intron inclusion and exon exclusion, and permits branch point elucidation. Plant. Physiol. 121, 411 -418.

Lal, S.K. and Hannah, L.C. (2005) Helitrons contribute to the lack of gene colinearity observed in modern maize inbreds. Proc. Natl. Acad. Sci. U S A. 102, 9993-4. 
Lal, S.K. and Hannah, L.C. (2005) Plant genomes: massive changes of the maize genome are caused by Helitrons. Heredity. 95, 421-2.

Morgante, M., Brunner, S., Pea, G., Fengler, K., Zuccolo, A. and Rafalski, A. (2005) Gene duplication and exon shuffling by Helitron-like transposons generate intraspecies diversity in maize. Nat. Genet. 37, 997-1002.

Poulter, R.T., Goodwin, T.J. and Butler, M.I. (2003) Vertebrate helentrons and other novel Helitrons. Gene. 313, 201-12.

Pritham, E.J. and Feschotte, C. (2007) Massive amplification of rolling-circle transposons in the lineage of the bat Myotis lucifugus. Proc Natl Acad Sci USA.104, 1895-900.

Song, R. and Messing, J. (2003) Gene expresiion of a gene family in maize based on noncollinear haplotypes. Proc. Natl. Acad. Sci. USA. 100, 9055-9060

Tavakoli, N., Comanducci, A., Dodd, H.M., Lett, M.C. and Albiger Bennett, P. (2000) IS1294, a DNA element that transposes by RC transposition. Plasmid. 44, 66-84.

Wang, Q. and Dooner, H.K. (2006) Remarkable variation in maize genome structure inferred from haplotype diversity at the bz locus. Proc. Natl. Acad. Sci. U S A. 103, 17644-9.

Xu, J.H. and Messing, J. (2006) Maize haplotype with a Helitron-amplified cytidine deaminase gene copy. BMC. Genet. 7, 52.

Zabala, G. and Vodkin, L.O. (2005) The wp mutation of Glycine max carries a gene-fragmentrich transposon of the CACTA superfamily. Plant. Cell. 17, 2619-32.

Zalaba ,G. and Vodkin, L.O. (2007) Novel exon combinations generated by alternative splicing of gene fragments mobilized by a CACTA transposon in Glycine max. BMC. Plant Biol.7, 38 